\title{
LA FRASE SCISSA. UN COSTRUTTO MARCATO NELL'INSEGNAMENTO DELL'ITALIANO A STRANIERI
}

di Stefania Gavazzi

\begin{abstract}
Le costruzioni sintatticamente marcate, caratterizzate cioè da un ordine dei costituenti diverso da quello canonico, hanno acquisito un'importanza crescente nella didattica dell'italiano a stranieri, tanto da essere trattate anche in alcuni manuali. Tra questi la frase scissa (FS) merita un'attenzione particolare fin dai primi livelli di un corso data la sua frequenza nel parlato e nello scritto e, più in generale, affinché gli studenti non madrelingua siano consapevoli delle diverse varietà linguistiche e combinatorie della lingua. Per questo è importante impiegare in classe testi autentici, brevi e concisi che permettano il focus immediato sulla forma da analizzare.
\end{abstract}

Keywords: Lingua italiana. Didattica dell'italiano. Varietà linguistiche.

\section{Resumo}

As construções sintaticamente marcadas, isto é, caracterizadas por uma ordem dos constituintes que divergem da norma, adquiriram crescente importância na didática de italiano para estrangeiros a ponto de figurarem em alguns manuais. Entre essas, o deslocamento da frase merece atenção particular já desde o início dos níveis iniciais de um curso devido a sua frequência tanto na modalidade oral da língua quanto na escrita e, de um modo mais geral, para que os alunos que estão aprendendo uma língua estrangeira possam conhecer as diversas variedades linguísticas e combinatórias da língua que estão aprendendo. Por esse motivo é importante que na sala de aula sejam usados textos autênticos, breves e concisos que permitam observar imediatamente as formas a serem analisadas.

Palavras-chave: Língua italiana. Didática da língua italiana. Variedades linguísticas.

\section{Considerazioni generali}

L'italiano viene generalmente considerato una lingua di tipo SVO, una lingua che prevede una sequenza degli elementi caratterizzata dalla sequenza Soggetto-VerboOggetto. Quest'ordine basico gode di un certo grado di flessibilità e può essere variato per avere costrutti sintatticamente marcati, i quali, sovvertendo la normale collocazione frastica SVO, consentono di conferire maggior rilievo a certi elementi della frase.

Tra i fenomeni più interessanti della sintassi marcata vi è la frase scissa (FS), una costruzione che risulta dalla suddivisione di una frase semplice SVO in due parti. La 
prima parte viene introdotta dal verbo essere e isola l'elemento messo in rilievo presentandolo come il focus dell'informazione, la seconda parte è collegata alla prima tramite "che" con funzione di generico subordinatore invariabile. Si veda l'esempio:

SVO

Il mio vicino fa molto rumore $\rightarrow$

\section{FRASE SCISSA}

$\mathbf{E}^{\prime}$ il mio vicino che fa molto rumore

Dal punto di vista informativo le due frasi sono equivalenti perché veicolano lo stesso messaggio. La FS però, non solo ha caratteristiche formali diverse rispetto alla frase SVO, possiede anche un valore aggiunto dal punto di vista pragmatico, una maggiore forza illocutiva, perché sposta tutto il peso informativo sul segmento frasale "il mio vicino" su cui si focalizza l'attenzione e questo risulta essere l'informazione nuova (il rema) da enfatizzare, mentre la subordinata "che fa molto rumore", è il contenuto presupposto (il tema) e pertanto conosciuto nello scambio comunicativo. $E^{\prime}$ una strategia sintattica volta ad evidenziare "il punto di maggiore salienza comunicativa della frase, l'elemento su cui si concentra maggiormente l'interesse del parlante e che fornisce la massima quantità di informazione nuova" (Berruto, 1987:77). Una struttura non marcata, al contrario, risulta più ovvia sia a livello dell'informazione che dell'interpretazione, di conseguenza l'impatto comunicativo sul destinatario è più debole.

La subordinata che segue l'elemento focalizzato (o rema) può essere di due tipi a) esplicita che + verbo al modo finito o $b$ ) implicita $a+$ verbo all'infinito:

SVO

Tu non capisci

\section{FRASE SCISSA}

a) Sei tu che non capisci (esplicita)

b) Sei tu $a$ non capire (implicita)

Una particolare tipologia di FS è rappresentata dalla pseudoscissa c) e d) che si differenzia dalla precedente per il fatto che la parte di testo contenente il verbo "essere" cioè la porzione di enunciato con la maggiore densità comunicativa, viene collocata dopo il subordinatore che ed è posta in fondo alla frase. 
$\begin{array}{lll}\text { Tu non capisci } & \rightarrow & \text { c) Quello che non capisce sei } \underline{\text { tu! }}\end{array}$

La FS rappresenta una delle tante modalità di realizzazione di rilievo informativo e, contrariamente a quanto si possa credere, non si tratta di un fenomeno innovativo tipico del neo standard. E' stata a lungo censurata o poco considerata dalla grammatica normativa perché ritenuta un francesismo molto in voga a partire dal Settecento, anche se qualche esempio era già presente nella letteratura italiana del Duecento e del Trecento, ma oggi è una struttura largamente presente nell'italiano sia orale che scritto.

Secondo gli studi di Roggia (2006) non è tratto esclusivo dell'oralità spontanea, la sua presenza si concentra piuttosto in situazioni comunicative più formali che necessitano un maggiore controllo dell'articolazione testuale e dunque molto vicine alla scrittura: ad esempio nel parlato-scritto dei telegiornali, dei radiogiornali e di certi programmi televisivi (litaliano trasmesso), e anche nel linguaggio della carta stampata (dove si registra una forte presenza della FS specialmente nella forma implicita), oppure in situazioni di parlato mono-direzionale come monologhi, conferenze, arringhe processuali, lezioni accademiche. In tutti i casi si tratta di circostanze contraddistinte da una forte asimmetria dei ruoli emittente-ricevente perché manca una reale dialettica fra le parti e che richiedono una maggiore pianificazione del discorso unitamente all'impiego di adeguate strategie per attirare l'attenzione dei destinatari sul focus informativo.

Anche se per gli studi di Roggia (2006) la presenza della FS negli scambi comunicativi del parlato spontaneo è piuttosto modesta, visto che questo possiede altre strategie di messa in rilievo come le dislocazioni o la semplice prosodia, esiste comunque un ampio spettro di espressioni rituali o convenzionali con presenza di FS, ad esempio "Com'è che non hai telefonato?" al posto del più classico "Perché non hai telefonato"? Oppure altre che sono fortemente legate alla comunicazione quotidiana dove domina l'immediatezza del messaggio. E' il caso delle FS con valore temporale ( $e$ ed $f$ ), interrogativo $(g$ ed $h)$ e contrastivo ( $i$ ed $l$ ) 
Non lo vedo da dieci anni

Ti aspetto da un' ora

Chi ha comprato il vino?

Quando parti?

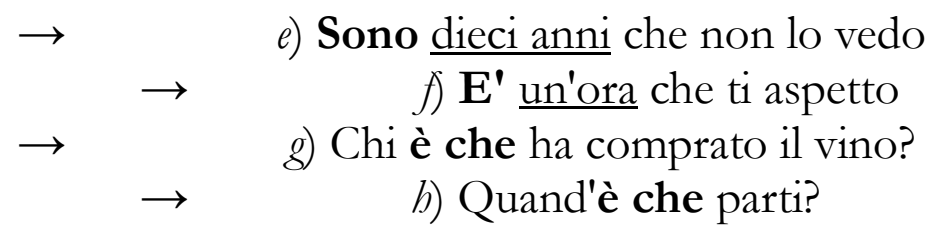

b) Quand'è che parti?

Si veda anche il seguente scambio comunicativo frequente nel parlato-parlato:

i) A. Hai preso le chiavi di casa?

b) A. Ho saputo che Roberto si sposa...
B. Eri tu che dovevi prenderle!

B. No, è suo fratello che si sposa!

In i) ed l) la presenza di FS nelle risposte di B segnala che quanto affermato da A non è corretto e deve essere rettificato o smentito: in questo caso la FS assume una funzione contrastiva.

In tutti gli esempi sopra citati il maggior grado di densità comunicativa non è fornito solo dalla diversa distribuzione degli elementi nella frase, ma in particolare dalla prosodia, cioè da una diversa distribuzione dell'accento rispetto ad una frase SVO.

\section{La frase scissa nella didattica dell'italiano L2/LS}

Alcuni costrutti sintatticamente marcati come le dislocazioni a sinistra/a destra e la frase scissa hanno già trovato spazio in qualche manuale ${ }^{1}$ e nella didassi della lingua italiana a stranieri. Il loro inserimento in un corso di italiano come L2/LS ha l'obiettivo di far riconoscere la variazione linguistica a seconda della tipologia testuale e della situazione comunicativa avvicinando i discenti alle diverse possibilità combinatorie del repertorio linguistico italiano attuale e di portarli così a conoscenza della vivacità e della varietà della lingua d'uso. Se, dunque, l'obiettivo di un corso di italiano è il raggiungimento di una competenza linguistico-comunicativa che non si limiti esclusivamente all'assimilazione di strutture e lessico, ma che faciliti la capacità di interagire con gli altri, la presentazione della FS comporta un triplice vantaggio: l'opportunità di scoprire la diversa disposizione dell'ordine delle parole secondo il loro

1 "Magari” edizioni Alma "Viaggio nell'italiano" edizioni Loescher e il Progetto "Lingua e Cittadinanza 20102011” livello B2 dell'Università per Stranieri di Siena visibile sul sito www.unistrasi.it 
valore informativo e di familiarizzare con una costruzione che ha una funzione tipicamente pragmalinguistica, nonché la possibilità di attirare l'attenzione su un altro aspetto del parlato: il ruolo dell'intonazione nella produzione di un costrutto sintattico marcato e della diversa organizzazione accentuale rispetto ad una frase SVO. La giusta intonazione, un aspetto paralinguistico essenziale nella dinamica conversazionale, ma a torto ritenuto una specie di sottocompetenza, e che spesso è assente anche nelle interlingue degli studenti di livello più avanzato, riveste, infatti, un ruolo determinante nello scambio comunicativo perché i parlanti nativi, evidenziando in modo diverso il focus dell'enunciato, permettono ai destinatari la corretta decodifica del messaggio.

Data la sua complessità strutturale e funzionale la FS merita una riflessione molto accurata fin dai livelli più elementari di un corso e non solo a livello sintattico, ma anche a livello pragmatico e paralinguistico, affinché la sua funzione nella lingua viva possa essere identificata e compresa. E' indispensabile inserirla in un contesto d'uso linguisticocomunicativo ben definito attraverso la presentazione di varie tipologie di testi autentici (scritti, audio, video), che siano funzionali alla pratica didattica e che tengano conto della dimensione reale della lingua. $\mathrm{E}^{\prime}$ necessario altresì che siano testi brevi con un input comprensibile e che permettano il focus immediato sulla forma da analizzare collegandola ad esperienze comunicative reali.

\section{Un'ipotesi di lavoro}

Di seguito viene illustrata un'ipotesi di lavoro su un breve testo pubblicitario autentico (in originale accompagnato dalla foto del prodotto) tratto da una rivista di largo consumo, che presenta un esempio di FS implicita. La scansione proposta permette di lavorare inizialmente sull'analisi di un testo scritto, per passare ad una fase strutturata in esercizi di fissaggio, per giungere, infine, ad una fase più autonoma che porta gli studenti all'elaborazione di una produzione scritta o orale. ${ }^{2}$

2 Queste sequenze di lavoro possono essere proposte a partire dal livello B1 o B1 potenziato. Spetta all'insegnante decidere il momento opportuno e le diverse modalità organizzative della classe (a gruppi, a coppie) per conferire alla didattica una dimensione più o meno ludica, più o meno coinvolgente. 
Nuovo Ava Pulito Naturale con Saponaria

Sono gli estratti naturali della pianta di Saponaria combinati con le scaglie di sapone a dar vita alla esclusiva formula di Nuovo Ava Pulito Naturale con Saponaria, un detersivo liquido per lavatrice in grado di regalare un pulito impeccabile e profondo nel totale rispetto di fibre e tessuti.

Fase1. Mostrare solo la foto del prodotto oppure portare in classe la confezione del prodotto originale. Farla descrivere e commentare liberamente dagli studenti. In particolare farli soffermare sul tipo di prodotto e sul suo uso, sulle parole, sui disegni, sui colori e annotare alla lavagna tutto quello che viene detto.

Fase 2. Distribuire il testo pubblicitario e farlo leggere lasciando il tempo per eventuali domande e chiarimenti richiesti dagli studenti.

Fase 3. Sottolineare la particolare costruzione della frase scissa evidenziando la differenza a livello sintattico e pragmatico tra il costrutto non marcato e quello marcato spiegando che con questo espediente linguistico si porta in evidenza la parte su cui si vuole attirare l'attenzione "gli estratti naturali di saponaria" che rappresentano l'informazione nuova (la vera novità del prodotto) su cui si deve concentrare tutta l'attenzione del potenziale consumatore.

SVO

Gli estratti naturali di saponaria danno

di saponaria vita a Nuovo Ava

Nuovo Ava
FRASE SCISSA

$\underline{\text { Sono gli estratti naturali }}$

a dar vita a

Aggiungere anche le due possibili realizzazioni della frase scissa:

Sono gli estratti naturali di saponaria a dar vita a Nuovo Ava (implicita)

Sono gli estratti naturali di saponaria che danno vita a Nuovo Ava (esplicita)

Fase 4. Presentare nuovi campioni di testi tratti da sequenze video o audio. Invitare gli studenti a individuare, trascrivere ed analizzare gli esempi di FS, chiedendo di focalizzare l'attenzione sulle curve intonative.

Fase 5. Distribuire alla classe esercizi di fissaggio. In particolare esercizi di 
a) trasformazione da frase non marcata a frase scissa (esplicita ed implicita) Il cellulare non funziona più $\quad \rightarrow \quad E^{\prime}$ il cellulare che non funziona più $E^{\prime}$ il cellulare a non funzionare più

b) formazione frasi non marcate e poi frasi scisse con le parole date in / bambini / giocare / $i$ / giardino $\rightarrow$ I bambini giocano in giardino Sono i bambini che giocano in giardino

c) trasformazione di domande in frasi scisse

Chi ba telefonato? $\quad \rightarrow \quad$ Chi è che ba telefonato?

d) trasformazione di frasi temporali in frasi scisse

Non vado al cinema da anni $\quad \rightarrow \quad$ Sono anni che non vado al cinema

Fase 6. Distribuire la foto di un nuovo prodotto o di un oggetto e far costruire un breve testo pubblicitario che contenga almeno un esempio di frase scissa.

Fase 7. Lettura e commento in plenum degli elaborati.

\section{Variante}

La fase 6 può essere modificata chiedendo agli studenti di elaborare uno spot pubblicitario o una telepromozione passando dallo stile impersonale e "tecnico" che contraddistingue il testo di partenza a uno più coinvolgente e personale cercando di creare una situazione comunicativa divertente e ironica. Nell'elaborazione del nuovo testo gli studenti hanno il compito di impiegare almeno uno o più esempi di FS.

\section{Conclusioni}

Quanto scritto rappresenta solo una breve traccia di un tema molto articolato e complesso come la frase scissa. Si inserisce tuttavia in una riflessione di più ampio respiro: vuole sottolineare l'importanza di una pratica didattica che sia attenta alla dimensione reale della lingua per avvicinare gli studenti ai principali aspetti del repertorio sociolinguistico italiano con l'intento di portarli ad una piena consapevolezza linguistica e ad una maggiore efficacia comunicativa. 


\section{Bibliografia}

Andorno C., 1999, Dalla grammatica alla linguistica. Basi per uno studio dell'italiano, Torino, Paravia scriptorium.

Bazzanella C., 1994, Le facce del parlare. Un approccio pragmatico dell'italiano parlato, Firenze, La Nuova Italia.

Berruto G., 1987, Sociolinguistica dell'italiano contemporaneo, Firenze, La Nuova Italia.

Coveri L., Benucci A., Diadori P., 1998, Le varietà dell'italiano. Manuale di sociolinguistica, Roma, Bonacci.

De Mauro T., (a c. di) 1994, Come parlano gli italiani, Firenze, La Nuova Italia.

Frison L., 1991, Le frasi scisse, in Renzi L., Salvi G., Cardinaletti A., (a c. di), Grande grammatica italiana di consultazione, Bologna, Il Mulino, vol. 1, pp.194-225.

Roggia C. E., 2006a, Costruzioni marcate tra scritto e parlato: la frase scissa, in Ferrari A. (a c. di), Parole frasi testi tra scritto e parlato, Cenobio LV/3 Rivista trimestrale di cultura, pp. 222-230.

Roggia C.E., 2006b, Frasi scisse (e altre costruzioni marcate) nella storia dellitaliano: alcune osservazioni, in A. Ferrari (a c. di), Parole frasi testi tra scritto e parlato, Cenobio LV/3 Rivista trimestrale di cultura, pp. 269-276.

Roggia C. E., 2008, "Frasi Scisse in italiano e in francese orale: evidenze dal C-ORAL$\mathrm{ROM}^{*}$ " in "Cuadernos de Filología Italiana", vol. 15, pp. 9-29 